Revista de Investigación de Física 23(1), (Ene-Jun 2020)

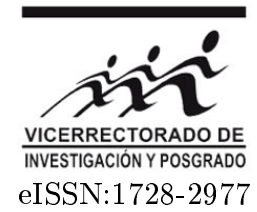

\title{
Estimación de la velocidad del agua en la Bahía Interior de Puno en el Lago Titicaca, mediante simulación numérica
}

\author{
César Aguirre*1, Julio Chicana ${ }^{2}$ y Marco Merma ${ }^{1}$ \\ ${ }^{1}$ Universidad Nacional Mayor de San Marcos, Lima, Perú \\ ${ }^{2}$ Universidad Nacional del Callao, Callao, Perú
}

Recibido 02 febrero 2020 - Aceptado 03 marzo 2020

\begin{abstract}
Resumen
La Bahía Interior de Puno, está ubicada en el Lago Titicaca al sur del Perú, de $2.6 \mathrm{~m}$ de profundidad media, $16.0 \mathrm{~km}^{2}$ de área superficial. La boca de la bahía, poblado de cañaverales nativos, se une a la Bahía Exterior por los canales norte y sur. La bahía, es un ejemplo del impacto negativo del humano, donde descargan agua residual tratado deficientemente, produciendo un incremento de biomasa algal, proceso de eutrofización y anoxia. El objetivo, fue estimar la dirección y magnitud de la velocidad de la corriente de agua, a escala horaria controlado por procesos físicos relevantes, entre el 23 y 30 de enero del 2019. Recogimos datos meteorológicos, profundidad y temperatura del agua. Realizamos experimentos computacionales usando el modelo para lagos ELCOM. El primer escenario consideró la bahía cerrada, y como resultado presentó flujo rotacional antihorario en el norte y horario en el sur, con rapidez que varió entre 0.5 y $4.0 \mathrm{~cm} / \mathrm{s}$. El segundo escenario, consideró ingreso y salida de agua por el canal norte y sur respectivamente, observándose un flujo del canal norte al canal sur, con rapidez que varió entre 20.0 y $50.0 \mathrm{~cm} / \mathrm{s}$
\end{abstract}

Palabras clave: Velocidad del agua, temperatura, viento, modelo computacional, eutrofización.

\section{Estimation of the speed of the water in the Inner Bay of Puno in Lake Titicaca, by means of numerical simulation}

\begin{abstract}
The Inner Bay of Puno is located on Lake Titicaca in southern Peru, $2.6 \mathrm{~m}$ in average depth, $16.0 \mathrm{~km}^{2}$ in surface area. The mouth of the bay, populated by native reed beds, joins the Outer Bay through the north and south channels. The bay is an example of the negative impact of humans, where they discharge poorly treated wastewater, producing an increase in algal biomass, eutrophication and anoxia. The objective was to estimate the direction and magnitude of the speed of the water current, on an hourly scale controlled by relevant physical processes, between January 23 and 30, 2019. We collected meteorological data, depth and temperature of the water. We performed computational experiments using the ELCOM lake model. The first scenario considered the closed bay, and as a result presented counterclockwise rotational flow in the north and clockwise in the south, with a speed that varied between 0.5 and $4.0 \mathrm{~cm} / \mathrm{s}$. The second scenario considered the entry and exit of water through the north and south channels, respectively, observing a flow from the north channel to the south channel, with a speed that varied between 20.0 and $50.0 \mathrm{~cm} / \mathrm{s}$.
\end{abstract}

Keywords: Water speed, temperature, wind, computational model, eutrophication.

\section{Introducción}

En regiones costeras y bahías, la calidad del agua es el resultado de procesos de mezcla, forzada principalmente por el viento, temperatura del aire, temperatura de afluentes, radiación solar y gradientes de presión derivado de la variación espacial de densidad. Entender la complejidad de zonas costeras y predecir con certidumbre el estado dinámico futuro, está limitado a la escasa capacidad de observación, así como falta de datos de pa- 
rámetros físicos recogidos de forma continua.

La Bahía Interior de Puno (BIP) está ubicado a $15^{\circ} 50^{\prime} 32^{\prime \prime}$ S y $69^{\circ} 59^{\prime} 42^{\prime \prime}$ O, en el Lago Titicaca (LT) al sur del Perú (Figura 1); de $2.6 \mathrm{~m}$ de profundidad media, $16.0 \mathrm{~km}^{2}$ de área superficial (Moreno, 2017) [Mor17], $41.0 \times 10^{6} \mathrm{~m}^{3}$ de volumen (Loza, 2016) [Loz16], y a 4000 msnm. La BIP es una región que se une a la Bahía Exterior de Puno (BEP) por dos canales, norte y sur de 20 y $50 \mathrm{~m}$ de ancho, rodeados de cañaverales nativos conocidos como totoras. Esta región, es la boca de la bahía de $4.5 \mathrm{~km}$ de longitud ubicado al este. Estos cañaverales funcionan como barreras naturales que restringen el intercambio de agua y sustancias con la BEP, que genera un débil intercambio de agua y sustancias a través de los canales (Figuras 1 y 3 ).

En regiones de intercambio restringido, existen fuertes evidencias del impacto humano. En la zona costera del Lago Titicaca, en particular, la BIP, la contaminación por altas cargas de materia orgánica y nutrientes que ingresan con aguas residuales, conducen a graves problemas de eutrofización y anoxia (Northcote,1992) [Nor92]. La BIP recibe agua residual tratado deficientemente producido por más de cien mil habitantes de la ciudad de Puno, que descargan aproximadamente $3.65 \times 10^{4} \mathrm{~m}^{3}$ al año (Robert, 2010) [Rob10]. Se ha encontrado concentración de clorofila-a de hasta $25 \mu \mathrm{g} / \mathrm{l}$, típico de lagos eutrófico-a-hipertróficos, en las proximidades de la BIP.

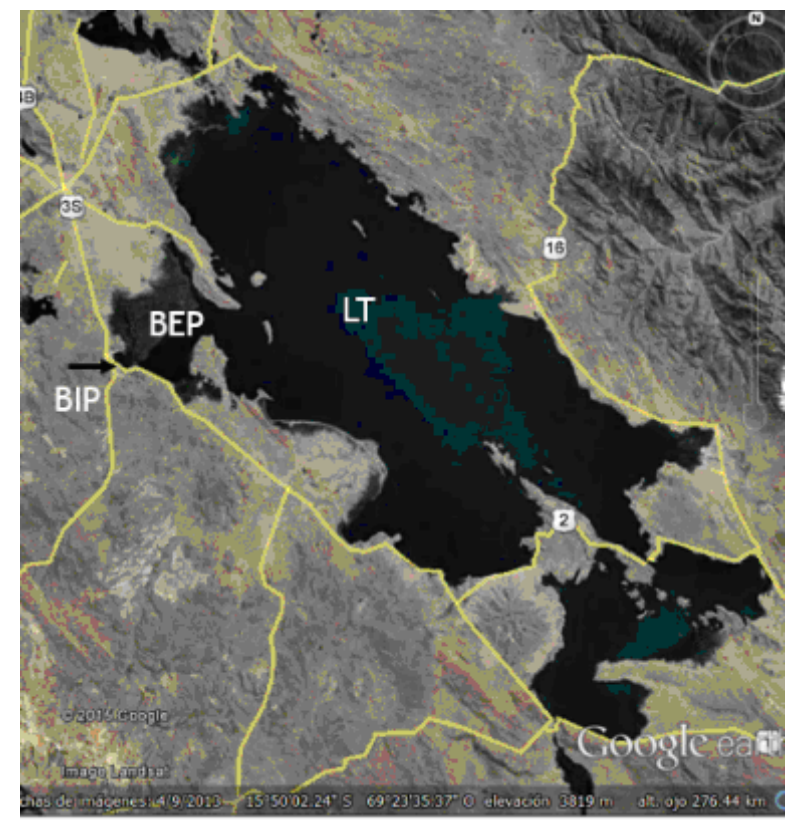

Figura 1: Lago Titicaca (LT). Bahía Exterior de Puno BEP. Bahía Interior de Puno BIP. Figura de Google Earth.

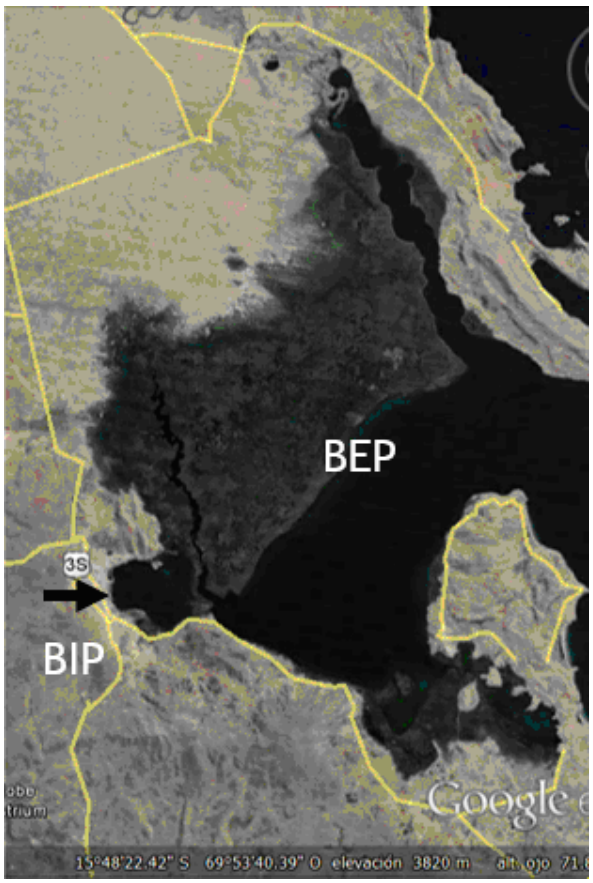

Figura 2: Bahía exterior de Puno (BEP). Bahía Interior de Puno (BIP). Figura de Google Earth.

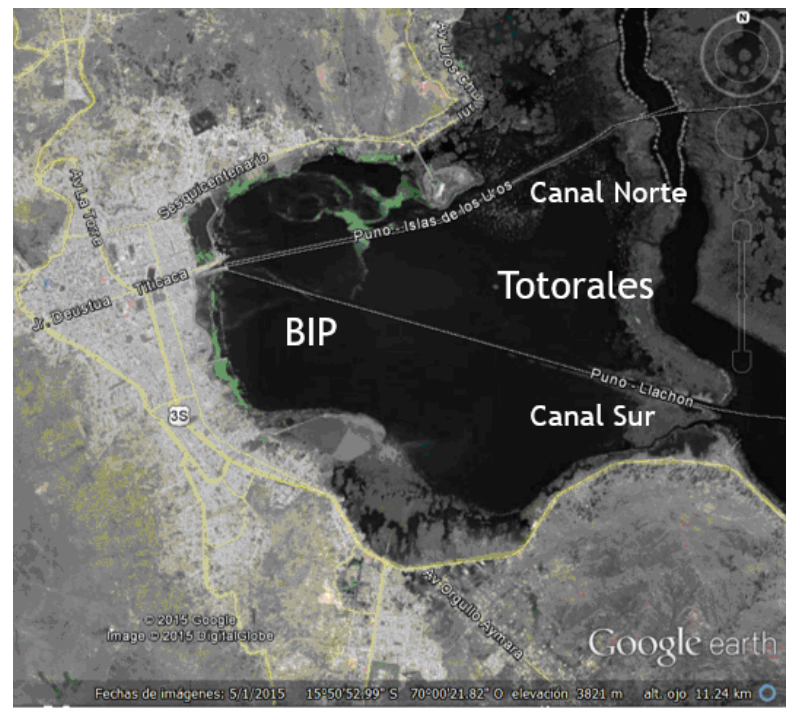

Figura 3: Bahía Interior de Puno (BIP). Canal norte y sur que conectan con la BEP rodeados de cañaverales denominados totoras, forman la boca de la BIP. Figura de Google Earth.

El crecimiento explosivo de la planta acuática lenteja de agua, que cubre la superficie del puerto de Puno, es la señal más clara de eutrofización de la bahía (Figura 4). El nivel de eutrofización de la bahía no puede ser entendido sólo en términos de cantidad de nutrientes y carga 
orgánica. También está estrechamente vinculado al débil intercambio de agua y sustancias que existe entre la BIP y la BEP. En particular en la BIP, existe poca información científica sobre este tipo de intercambio y de los procesos físicos que controlan el flujo de agua.

El objetivo, fue estimar la dirección y magnitud de la velocidad de la corriente de agua en la BIP a escala horaria y diaria, controlado por procesos físicos relevantes, mediante simulación numérica.

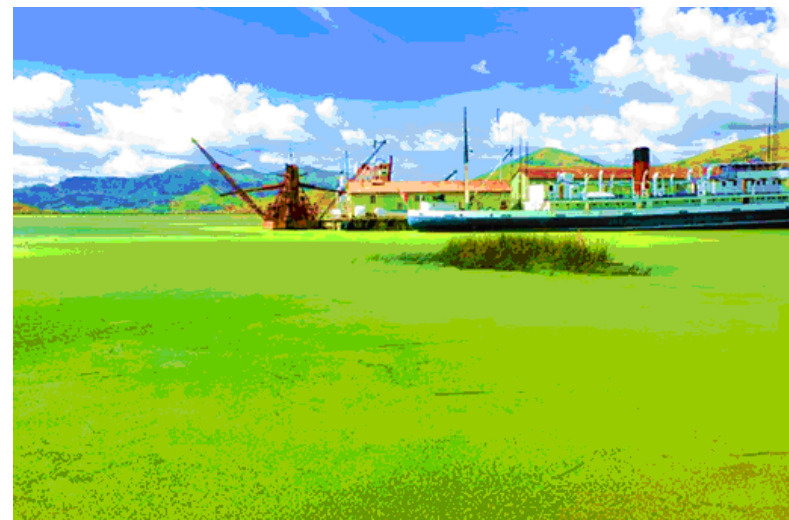

Figura 4: Puerto de Puno, el agua de la bahía se cubre por una capa de algas denominados lenteja de agua, producto del exceso de nutrientes. Fuente: https://www.google.com/ search?q=fotos $+d e+$ lenteja.

El periodo de estudio fue entre el 23 y 30 de enero del 2019. Consideramos entre los procesos físicos que controlan el intercambio horizontal, los forzantes meteorológicos, la topografía y la temperatura del agua de la bahía. Para cumplir con el objetivo se utilizó una combinación de argumentos de escala, en base a datos de campo exis- tentes, así como de datos de campo adicionales recogidos en el periodo de estudio, y un modelo computacional para lagos, bahías, presas y estuarios ELCOM, que permitió realizar simulaciones en dos escenarios, (Hodges, 2000) [Hod00] , (Hodges,2006) [Hod06] . Antecedentes de mediciones de la velocidad del flujo en la Bahía Interior mediante simulación numérica, fue realizado por (Huiza,2011), elaboró un modelo propio desarrollado en Matlab, y aplico a la bahía. Validó sus resultados con datos medidos en mayo del 2002 por el Proyecto Especial del Lago Titicaca PELT. Considero valores de rapidez del viento de $9.0 \mathrm{~m} / \mathrm{s}$, mientras que en el presente trabajo está en el orden de $3.0 \mathrm{~m} / \mathrm{s}$ [Hui11].

\section{Metodología}

\section{Datos medidos en la BIP}

a. Los registros meteorológicos fueron obtenidos de una estación meteorológica propia, instalado en el puerto de Puno, a $15 \mathrm{~m}$ de altura, configurado para medir datos de magnitud y dirección del viento, temperatura del aire, humedad relativa, radiación solar, y precipitación, cada 5 minutos del 23 al 30 de enero del 2019 (Figura 5).

b. En el mismo periodo, se obtuvo datos de profundidad en 47 puntos, usando un GPS, una wincha y un peso. Sin embargo, esta cantidad de puntos no fueron suficientes para interpolar a todo el dominio, por lo que se consideró usar datos existentes de batimetría del Lago Titicaca, que permitió obtener la batimetría de la BIP. Los datos de batimetría del LT fueron facilitados por la Dirección de Hidrografía y Navegación de la Marina de Guerra del Perú. Esta batimetría es una matriz de 71 filas por 66 columnas (Figura 6).

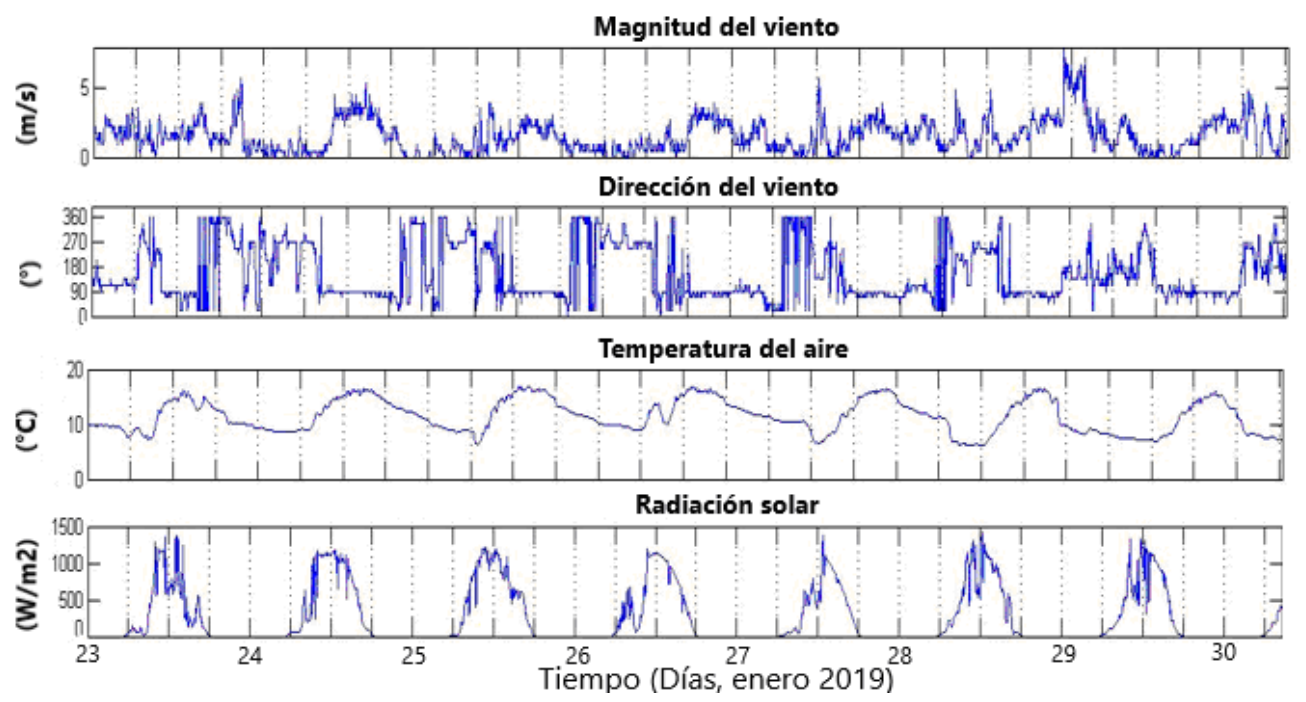

Figura 5: Datos meteorológicos recogidos en el puerto de Puno entre 23 y 30 de enero de 2019. 


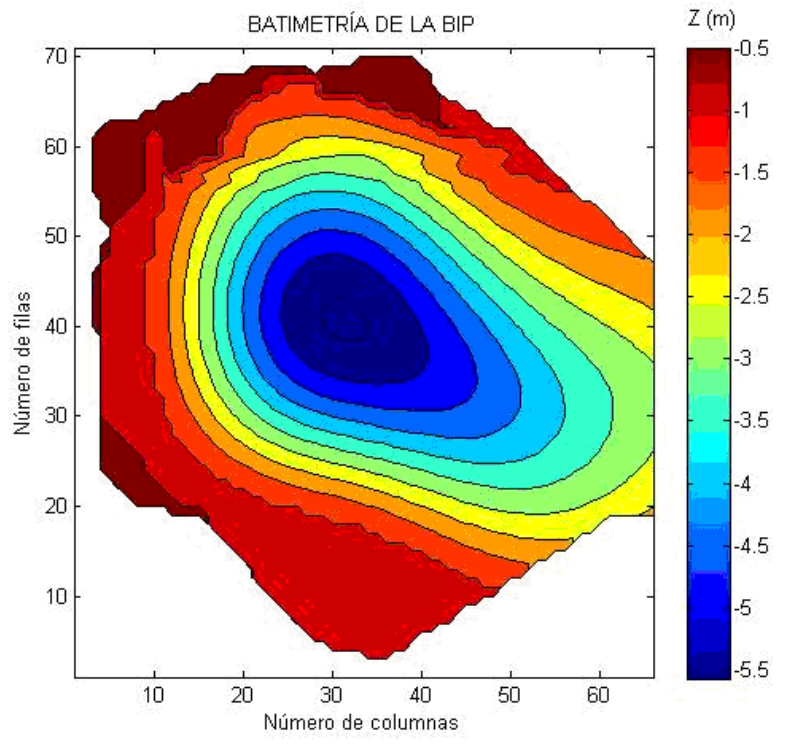

Figura 6: Batimetría de la BIP.

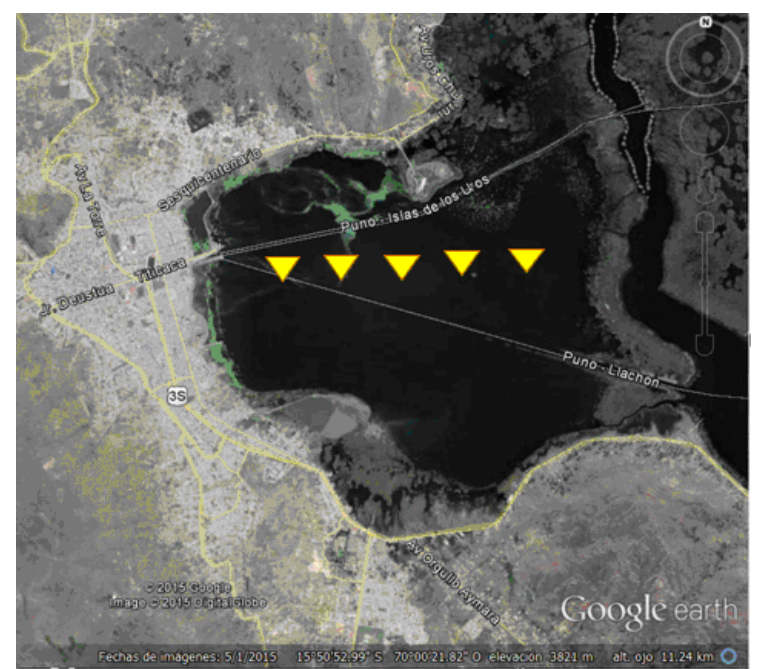

Figura 7: Posición de los perfiles de temperatura en la BIP. Figura de Google Earth.

\section{Simulaciones con ELCOM}

El modelo computacional para lagos, bahías, estuarios y presas ELCOM opera con dos carpetas Pre y Run.

a. Pre. Denominado preprocesamiento, requiere el archivo batimetría de la BIP. En el archivo se especificó la resolución espacial horizontal $\mathrm{dx}=\mathrm{dy}=80 \mathrm{~m}$, y vertical $\mathrm{dz}=0.5 \mathrm{~m}$ de 12 capas, además la latitud, altitud y la matriz de batimetría. En el archivo de condiciones de frontera, se consideró el primer escenario con la bahía cerrada sin ingreso ni salida de agua. En el segundo escenario, se abrió los canales norte y sur con ingreso y salida de agua de caudal variable entre 0 y $200 \mathrm{~m}^{3} / \mathrm{s}$ respectivamente.
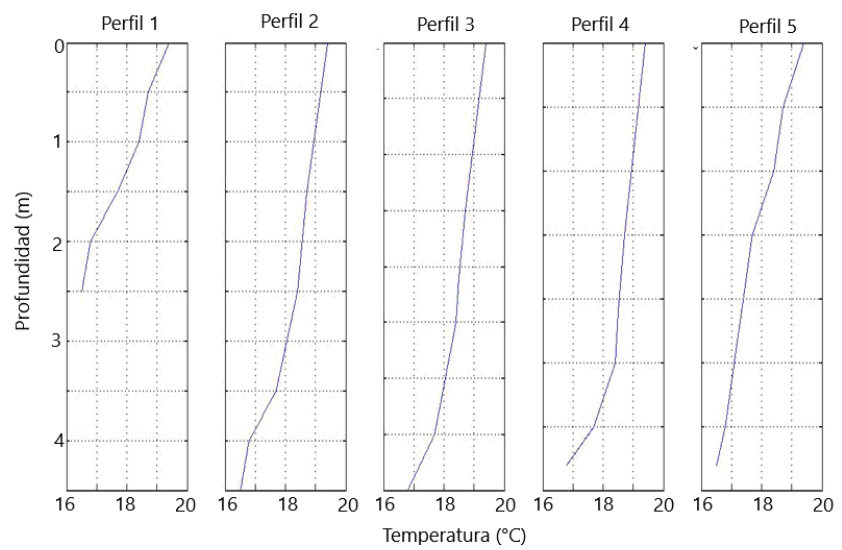

Figura 8: Perfiles de temperatura del agua en la BIP. Figura propia.

b. Run. Denominado post procesamiento, requiere el archivo de forzantes meteorológicos, perfiles de temperatura, caudal de ingreso y salida por los canales. Además, se configuró el modelo a condiciones de un fluido newtoniano, sin considerar la rotación de la Tierra. La salida del modelo numérico entregó campos espaciales horizontales y temporales de velocidad del agua, como promedios de las capas verticales, cada dos horas. Al correr el modelo, se realizó una serie de correcciones hasta encontrar el paso de tiempo adecuado para mantener dentro del rango, la velocidad de la onda barotrópica y baroclínica llegando a encontrar el paso de tiempo óptimo de $80 \mathrm{~s}$, el número de interacciones de 7500 , el tiempo total de simulación 168 h, fecha de inicio 23 de enero de 2019 a las 00:00 horas.

\section{Resultados}

En esta sección, presentamos la magnitud y la dirección de la velocidad promedio vertical, obtenidos por medio del modelo numérico en el plano horizontal de la bahía.

\section{Simulación S1}

\section{Primer escenario, dominio cerrado}

1-S1 26-enero-2019, 06:00 h. El centro de la bahía presentó flujo rotacional antihorario, con rapidez mínima y máxima de 0.5 y $5.0 \mathrm{~cm} / \mathrm{s}$; notar que en la mayor parte de la bahía el agua se desplazó con rapidez mínima. La intensidad del viento fue de $0.2 \mathrm{~m} / \mathrm{s}$, y viene del oeste. La radiación solar fue de $100 \mathrm{~W} / \mathrm{m}^{2}$ (Figura 9). 


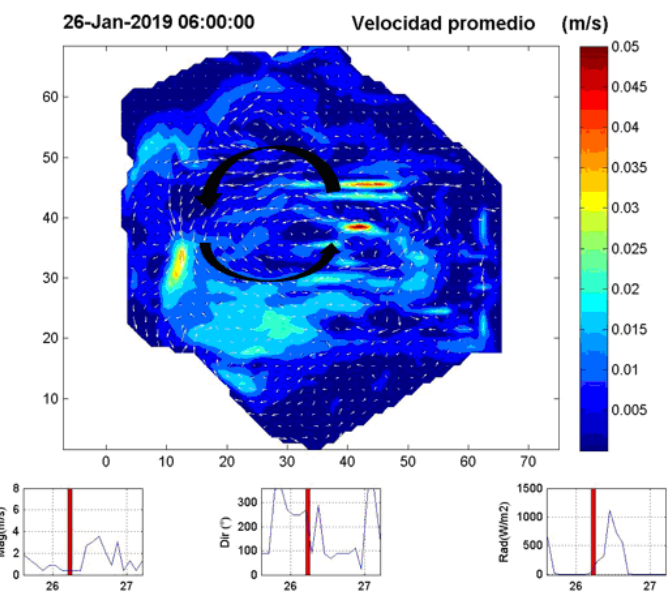

Figura 9: Campo de velocidad promedio vertical de la BIP, del 26 de enero de 2019, a las 06:00 h.

2-S1 26-enero-2019, 12:00 h. La bahía presentó dos flujos rotacionales, al norte antihorario, y al sur horario, con rapidez mínima y máxima de 0.5 y $3.5 \mathrm{~cm} / \mathrm{s}$. Notar que en grandes regiones de la bahía el agua se desplaza con velocidad de $1.5 \mathrm{~cm} / \mathrm{s}$. La intensidad del viento aumentó a $2.8 \mathrm{~m} / \mathrm{s}$ y viene del este. La radiación solar aumentó a $1000 \mathrm{~W} / \mathrm{m}^{2}$ (Figura 10).

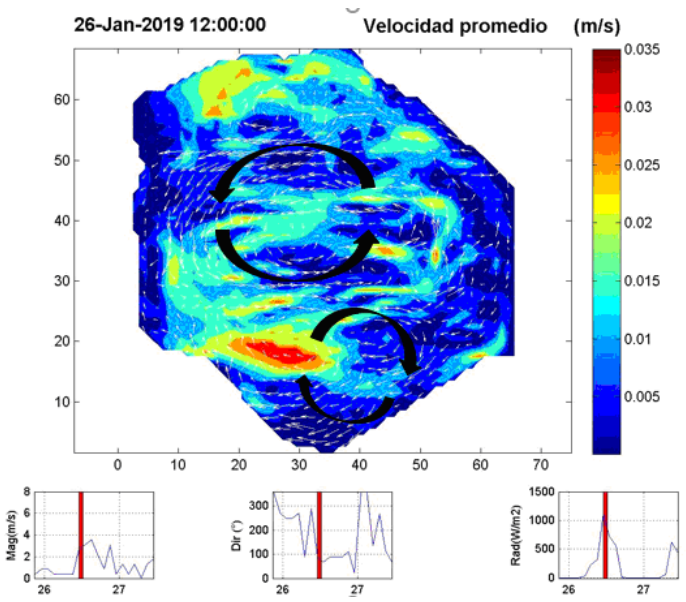

Figura 10: Campo de velocidad promedio vertical de la BIP, del 26 de enero de 2019, a las 12:00 h.

3-S1 26-enero-2019, 18:00 h. La bahía mantuvo los flujos rotacionales, al norte antihorario, y al sur horario, con rapidez mínima y máxima de 1.0 y 10.0 $\mathrm{cm} / \mathrm{s}$. Notamos que la rapidez máxima se presentó en pequeños sectores, siendo más notable regiones donde el agua se desplazó con rapidez de $4.0 \mathrm{~cm} / \mathrm{s}$. La intensidad del viento fue de $2.0 \mathrm{~m} / \mathrm{s}$, y viene del este. La radiación solar cayó a $0.0 \mathrm{~W} / \mathrm{m}^{2}$ (Figura 11).

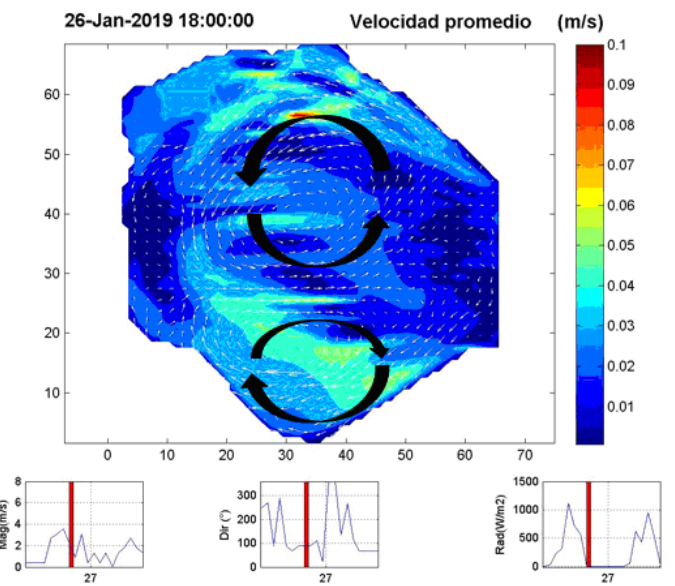

Figura 11: Campo de velocidad promedio vertical de la BIP, del 26 de enero de 2019, a las 18:00 h.

4-S1 27-enero-2019, 00:00 h. La bahía mantuvo los flujos rotacionales antihorario y horario como el caso anterior, aumentado la rapidez máxima a $8.0 \mathrm{~cm} / \mathrm{s}$ y mantuvo la mínima en $1.0 \mathrm{~cm} / \mathrm{s}$. Presentó grandes regiones de la bahía donde el agua se desplazó con rapidez de $4.0 \mathrm{~cm} / \mathrm{s}$. La intensidad del viento disminuyó a $1.0 \mathrm{~m} / \mathrm{s}$, y viene del norte. La radiación solar fue de $0.0 \mathrm{~W} / \mathrm{m}^{2}$ (Figura 12).

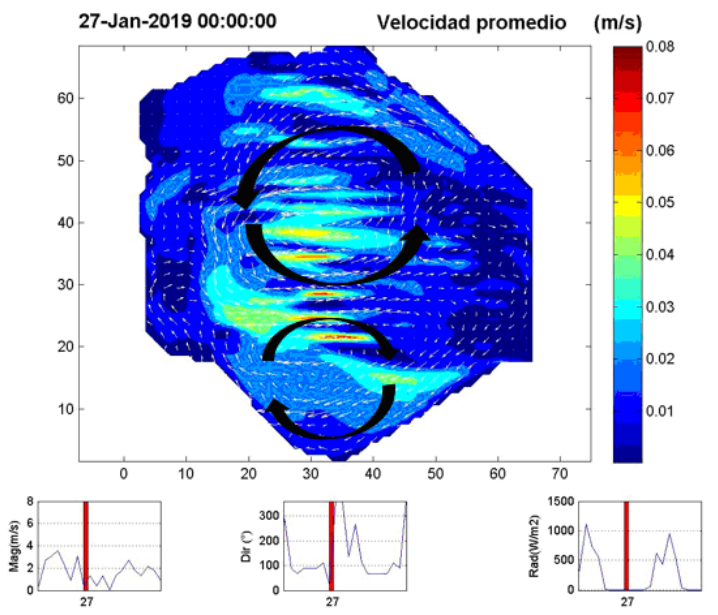

Figura 12: Campo de velocidad promedio vertical de la BIP, del 27 de enero de 2019, a las 00:00 h.

5-S1 27-enero-2019, 06:00 h. El flujo rotacional se desvaneció en toda la bahía generando una distribución aleatoria, disminuyendo la rapidez mínima y máxima a 0.5 y $4.0 \mathrm{~cm} / \mathrm{s}$. La intensidad del viento disminuyó notablemente a $0.1 \mathrm{~m} / \mathrm{s}$, y viene del oeste. La radiación solar se mantiene en $0.0 \mathrm{~W} / \mathrm{m}^{2}$ (Figura 13). 


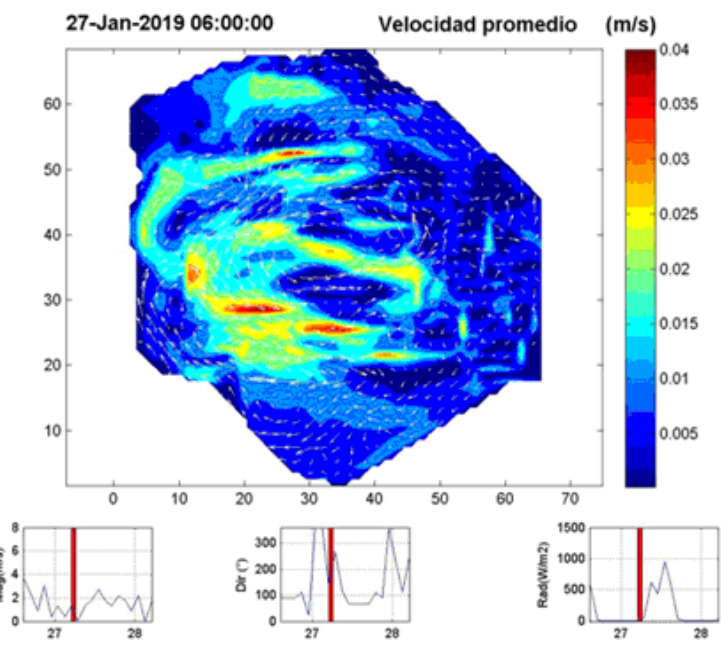

Figura 13: Campo de velocidad promedio vertical de la BIP, del 27 de enero de 2019, a las 06:00 h.

6-S1 27-enero-2019, 12:00 h. La bahía volvió al mismo patrón de circulación presentada en los casos anteriores, con dos flujos rotacionales al norte antihorario y sur horario. La rapidez máxima aumentó a $5.5 \mathrm{~cm} / \mathrm{s}$, y la mínima se mantuvo en $0.5 \mathrm{~cm} / \mathrm{s}$. Se incrementó la intensidad del viento a $2.0 \mathrm{~m} / \mathrm{s}$, y viene del este. La radiación solar fue de $1000 \mathrm{~W} / \mathrm{m}^{2}$ (Figura 14).

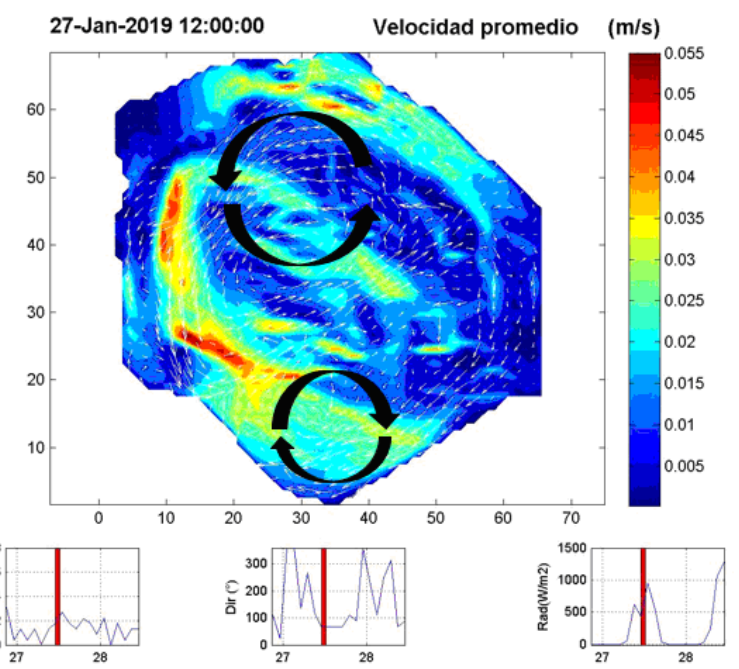

Figura 14: Campo de velocidad promedio vertical de la BIP, del 27 de enero de 2019, a las 12:00 h.

\section{Simulación S2}

Segundo escenario, dominio abierto en el canal norte y sur.

El segundo escenario, fue más cercano a la realidad, se abrieron los canales norte y sur que conectan con la bahía Exterior. Por el canal del norte ingresó agua a la bahía con un caudal variable de 0.0 a $200 \mathrm{~m}^{3} / \mathrm{s}$, por el canal del sur salió agua hacia la bahía exterior con caudal igual al anterior. Los valores variables del caudal fueron artificiales, debido a la falta de datos medidos en los canales en el periodo de estudio.

1-S2 26-enero-2019, 00:00 h. La bahía presentó en el centro un flujo rotacional antihorario, y rapidez mínima y máxima de 5.0 y $10.0 \mathrm{~cm} / \mathrm{s}$, valores superiores a los encontrados en la primera simulación numérica. Los resultados del modelo numérico muestran una rapidez mínima casi uniforme en toda la bahía. Por el canal del norte ingresó agua con un caudal de 50 $\mathrm{m}^{3} / \mathrm{s}$ hacia el oeste, por el canal del sur salió agua con caudal de $50 \mathrm{~m}^{3} / \mathrm{s}$ hacia el este. La intensidad del viento fue de $0.6 \mathrm{~m} / \mathrm{s}$, con dirección desde el oeste. La radiación solar fue de $0.0 \mathrm{~W} / \mathrm{m}^{2}$ (Figura 15).

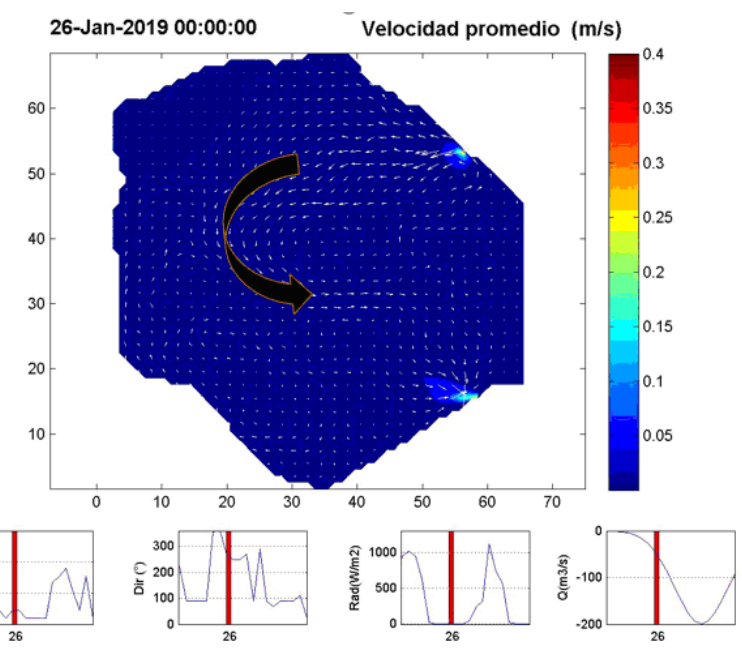

Figura 15: . Campo de velocidad promedio vertical de la BIP, del 26 de enero de 2019, a las 00:00 h. Segunda simulación.

2-S2 26-enero-2019, 06:00 h. La bahía mantuvo en el centro un flujo rotacional antihorario con trayectoria del canal norte hacia el canal sur, y se incrementaron los valores de rapidez mínima y máxima a 10.0 y $30.0 \mathrm{~cm} / \mathrm{s}$, valores superiores al caso anterior y a la primera simulación. Presentó rapidez mínima casi uniforme en toda la bahía a diferencia de las regiones donde la pluma de agua ingresó y salió, debido al incremento del caudal a 150 $\mathrm{m}^{3} / \mathrm{s}$. La intensidad del viento fue de $0.5 \mathrm{~m} / \mathrm{s}$, y viene del oeste. La radiación solar fue de $100.0 \mathrm{~W} / \mathrm{m}^{2}$ (Figura 16). 


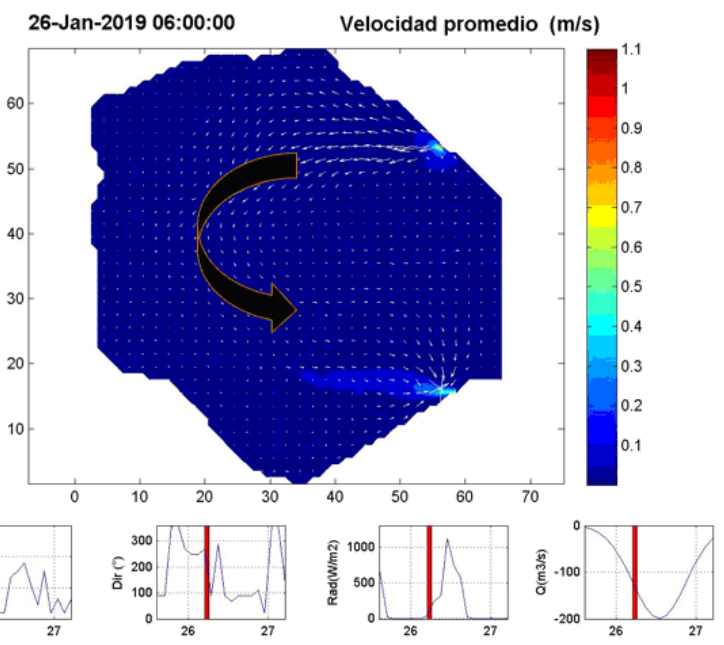

Figura 16: . Campo de velocidad promedio vertical de la BIP, del 26 de enero de 2019, a las 06:00 h. Segunda simulación.

3-S2 26-enero-2019, 12:00 h. La bahía mantuvo en el centro un flujo rotacional antihorario con trayectoria del canal norte hacia el canal sur, y se incrementaron los valores de la rapidez mínima y máxima a 20.0 y $50.0 \mathrm{~cm} / \mathrm{s}$, valores superiores al caso anterior y a la primera simulación, debido al incremento del caudal al valor máximo de $200.0 \mathrm{~m}^{3} / \mathrm{s}$. Mantuvo rapidez mínima casi uniforme en toda la bahía. La intensidad del viento aumentó a $2.2 \mathrm{~m} / \mathrm{s}$, y viene del este. La radiación solar fue de $1000.0 \mathrm{~W} / \mathrm{m}^{2}$ (Figura 17).

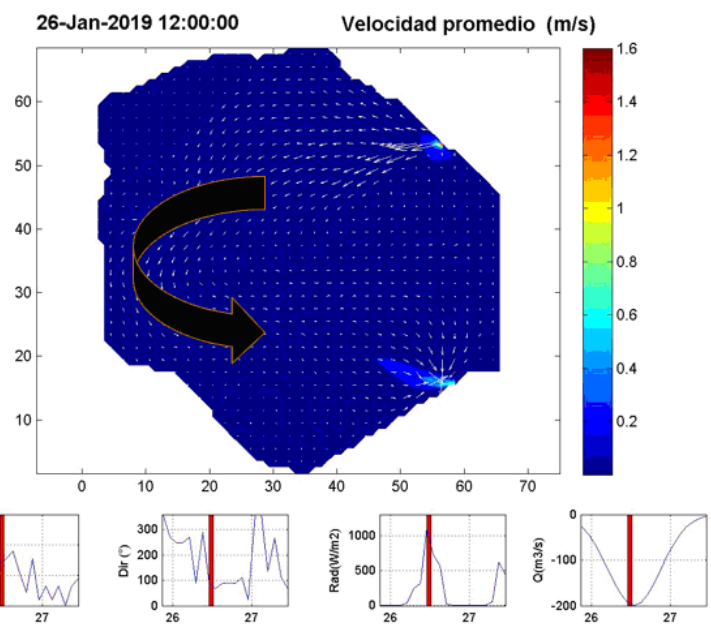

Figura 17: Campo de velocidad promedio vertical de la BIP, del 26 de enero de 2019, a las 12:00 h. Segunda simulación.

4-S2 26-enero-2019, 18:00 h. La bahía mantuvo el mismo patrón de circulación en el centro, con valores de rapidez mínima y máxima de 15.0 y $40.0 \mathrm{~cm} / \mathrm{s}$, menores al caso anterior. Presentó una rapidez mínima ca- si uniforme en toda la bahía a diferencia de las regiones por donde la pluma de agua ingresó y salió. El caudal empezó a disminuir a $160.0 \mathrm{~m}^{3} / \mathrm{s}$. La intensidad del viento disminuyó a $2.0 \mathrm{~m} / \mathrm{s}$, y viene del este. La radiación solar fue de $0.0 \mathrm{~W} / \mathrm{m}^{2}$ (Figura 18).

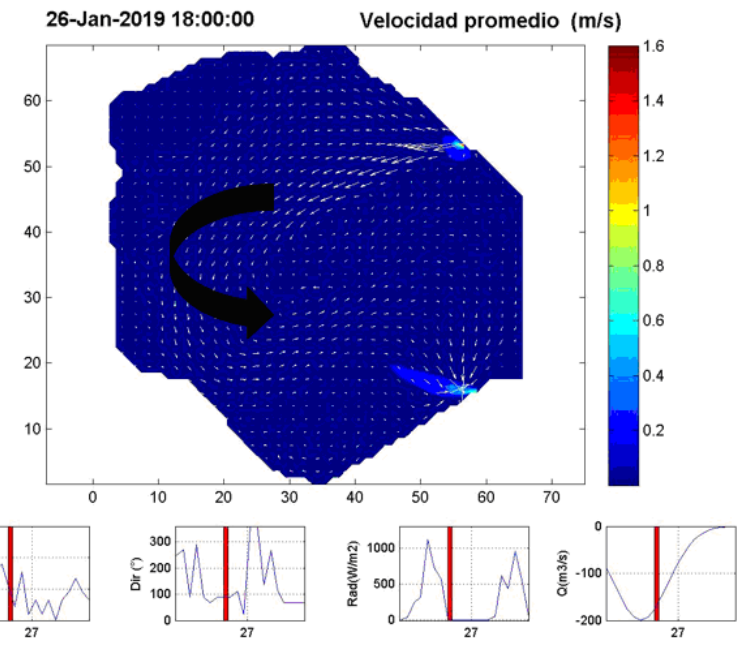

Figura 18: Campo de velocidad promedio vertical de la BIP, del 26 de enero de 2019, a las 18:00 h. Segunda simulación.

5-S2 27-enero-2019, 00:00 h. La circulación en la bahía se mantuvo como en las horas anteriores, con rapidez mínima y máxima de 10.0 y $30.0 \mathrm{~cm} / \mathrm{s}$. En la mayor parte de la bahía, el agua se desplazó con rapidez mínima. El caudal de ingreso y salida siguió disminuyendo a $100 \mathrm{~m}^{3} / \mathrm{s}$. La intensidad del viento disminuyó a $0.6 \mathrm{~m} / \mathrm{s}$, y viene del norte. La radiación solar fue de $0.0 \mathrm{~W} / \mathrm{m}^{2}$ (Figura 19).

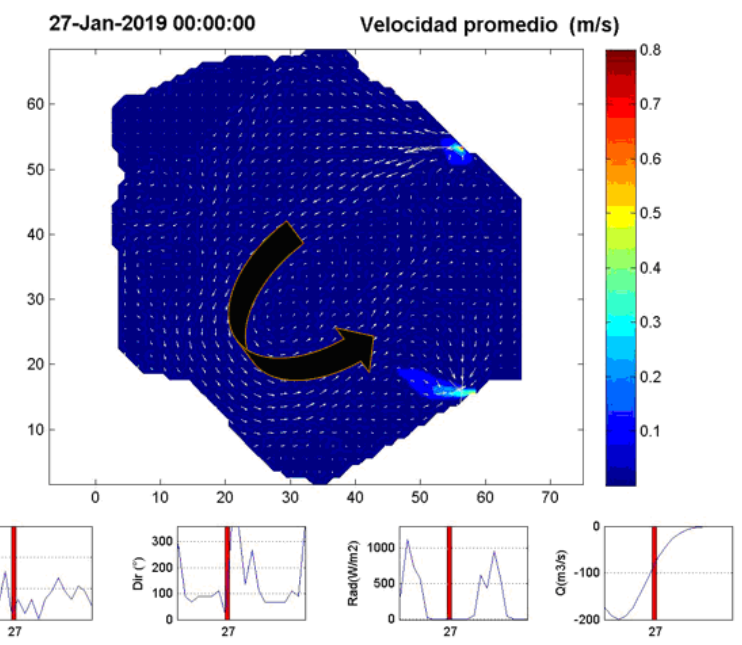

Figura 19: Campo de velocidad promedio vertical de la BIP, del 27 de enero de 2019, a las 00:00 h. Segunda simulación.

6-S2 27-enero-2019, 06:00 h. La circulación en la bahía presentó un flujo rotacional en toda la bahía, 
con disminución de la rapidez mínima y máxima a 5.0 y $10.0 \mathrm{~cm} / \mathrm{s}$. En la mayor parte de la bahía, el agua se desplazó con rapidez mínima. El caudal de ingreso y salida disminuyó $5.0 \mathrm{~m}^{3} / \mathrm{s}$. La intensidad del viento se mantiene en $0.6 \mathrm{~m} / \mathrm{s}$, y viene del oeste. La radiación solar fue de $0.0 \mathrm{~W} / \mathrm{m}^{2}$ (Figura 20).

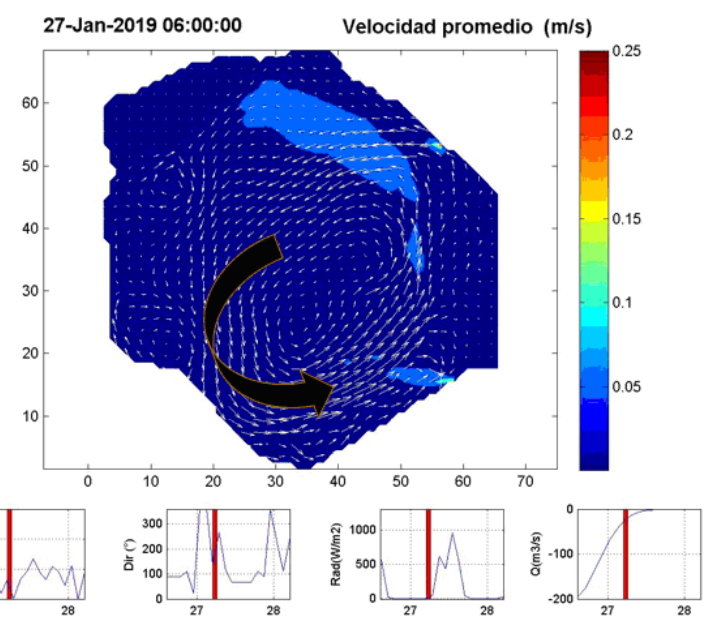

Figura 20: Campo de velocidad promedio vertical de la BIP, del 27 de enero de 2019, a las 06:00 h. Segunda simulación.

7-S2 27-enero-2019, 12:00 h. La circulación en la bahía presentó un flujo rotacional en toda la bahía, con disminución de la rapidez mínima y máxima a 0.5 y 5.5 $\mathrm{cm} / \mathrm{s}$. El patrón de circulación que presentó fue semejante a la primera simulación debido a la disminución del caudal de ingreso y salida al mínimo de $0.0 \mathrm{~m}^{3} / \mathrm{s}$. La intensidad del viento aumentó a $2.0 \mathrm{~m} / \mathrm{s}$, y viene del este. La radiación solar fue de $800.0 \mathrm{~W} / \mathrm{m}^{2}$ (Figura 21).

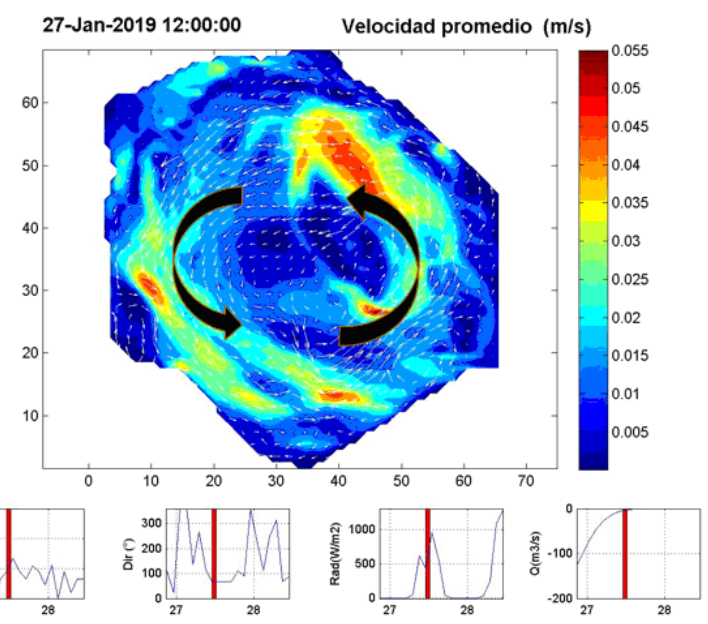

Figura 21: Campo de velocidad promedio vertical de la BIP, del 27 de enero de 2019, a las 12:00 h. Segunda simulación.

\section{Discusión}

\section{Datos meteorológicos}

La dirección del viento por lo general presentó tres tendencias, a media noche viene del norte, en la mañana, del oeste y por la tarde del este. La rapidez del viento presentó máximo y mínimo de 8.0 y $0.1 \mathrm{~m} / \mathrm{s}$, y promedio de $2.6 \mathrm{~m} / \mathrm{s}$.

La temperatura del aire presentó el ciclo diario, al calentarse al medio día hasta $15.0^{\circ} \mathrm{C}$ y por la noche disminuir hasta $8.0^{\circ} \mathrm{C}$.

La radiación solar presentó el ciclo diario, llegando a valores máximos al medio día de $1000.0 \mathrm{~W} / \mathrm{m}^{2}$.

La precipitación no fue muy notable en el periodo de estudio.

\section{Batimetría de la bahía}

La batimetría de la bahía no concuerda exactamente con la topografía real, debido a la interpolación realizada, parece un recipiente con un fondo en el centro de la bahía, de $5.5 \mathrm{~m}$ de profundidad máxima. La profundidad depende del nivel del lago, que varía según la estación del año. Huisa (2011) presentó $6.0 \mathrm{~m}$ de profundidad máxima y podría aumentar hasta $8.0 \mathrm{~m}$ en época de lluvias. También necesita mejorar la línea de costa.

\section{Simulación S1}

En el primer escenario, los flujos rotacionales presentados es la respuesta de la bahía a la topografía, y energía mecánica inducida por el viento. Solo en la simulación 5S1 no presentó flujo rotacional, debido a la disminución de la intensidad del viento a $0.1 \mathrm{~m} / \mathrm{s}$. Otro hallazgo importante fue el desplazamiento del agua en la bahía, con rapidez muy pequeña entre 0.5 y $4.0 \mathrm{~cm} / \mathrm{s}$. Estos valores son muy difíciles de detectar con correntómetros comunes por lo que es necesario equipo de alta sensibilidad.

\section{Simulación S2}

En el segundo escenario, al abrir el canal norte y sur con caudal variable entre 0.0 y $200.0 \mathrm{~m}^{3} / \mathrm{s}$, generó cambios notables en la dirección del flujo y rapidez de la corriente. La dirección del flujo se orientó del canal norte al canal sur de la bahía. Aumentó la rapidez mínima y máxima del agua a 20.0 y $50.0 \mathrm{~cm} / \mathrm{s}$, cuando el caudal alcanzó el valor máximo de $200.0 \mathrm{~m}^{3} / \mathrm{s}$. La intensidad del viento influyó muy poco en este patrón. En la simulación 7-S2, cuando el caudal llega $0.0 \mathrm{~m}^{3} / \mathrm{s}$, y la rapidez del viento fue de $2.0 \mathrm{~m} / \mathrm{s}$, el patrón de circulación vuelve a presentar las características del primer escenario S1.

La validación de los resultados no se pudo presentar, por falta de datos medidos de velocidad del agua en la bahía, canal norte y sur en la fecha de estudio. Sin embargo, comparando con rapidez mínima y máxima de 0.71 
y $5.3 \mathrm{~cm} / \mathrm{s}$ medidos por Huisa, 2011, a $60 \%$ de profundidad en la zona oeste de la bahía, fueron cercanos a los resultados entregados por ELCOM en el primer escenario [Hui11].

\section{Conclusiones}

La dirección de la velocidad de la corriente de agua en la bahía presentó en el primer escenario flujos rotacionales antihorarios en el norte y horarios en el sur, causados principalmente por la topografía de la bahía y la intensidad del viento.

La dirección de la velocidad de la corriente de agua, en el segundo escenario cambió notablemente, presentó un flujo rotacional orientado del canal norte (entrada) al canal sur (salida), debido al valor variable del caudal y la topografía de la bahía.

Respecto a la magnitud de la velocidad de la corriente de agua, es notable la diferencia entre los dos escenarios, encontrando valores mínimos y máximos muy diferentes, en el primer escenario de 0.5 y $4.0 \mathrm{~cm} / \mathrm{s}$ y en el segundo escenario de 20.0 y $50.0 \mathrm{~cm} / \mathrm{s}$.

También se encontró direcciones preferenciales del viento que se repiten a escala diaria, y de intensidad entre 0.0 y $3.0 \mathrm{~m} / \mathrm{s}$ que influyó en el patrón de circulación, manteniendo el flujo rotacional, y cuando disminuyó a $0.1 \mathrm{~m} / \mathrm{s}$ presentó un patrón de circulación completamen- te desordenando.

Respecto a la validación de los resultados, la simulación del primer escenario sería el mejor, comparando con resultados de rapidez mínima y máxima existentes. Sin embargo, no podemos dar estimaciones finales por las condiciones variables que presenta la dinámica de la bahía durante todo el año.

Queda por hacer, una batimetría más cercana a la realidad. Realizar mediciones de velocidad de la corriente de agua en la bahía, a diferentes profundidades y calcular la velocidad promedio, para validar los resultados del modelo. Medir el ancho, profundidad y velocidad del agua en el canal norte y sur, para estimar el caudal. Presentar campos de velocidad entregados por el modelo en la superficie y fondo de la bahía. Modelar la zona de totoras como frontera abierta, incrementado el coeficiente de arrastre.

\section{Agradecimientos}

A la Universidad Nacional Mayor de San Marcos, por su apoyo a través del proyecto B18131171 que sirvió para adquirir equipos y financiar la salida de campo. A la Dirección de Hidrografía y Navegación de la Marina de Guerra del Perú, por facilitar los datos de batimetría del Lago Titicaca. A los autores del modelo computacional ELCOM.

\section{Referencias}

[Hod00] Hodges, B. (2000). Numerical Techniques in CWR-ELCOM Water Research. University of Western Australia.

[Hod06] Hodges, B. y Dallimore, C. (2006). Estuary, Lake and Coastal Ocean Model: ELCOM. Manual Científico. University of Western Australia.

[Hui11] Huisa, D. (2011). Modelamiento matemático de la circulación del flujo de la bahía de Puno. Tesis de Maestría, Facultad de Ingeniería Civil, Universidad Nacional de Ingeniería, Lima Perú.

[Loz16] Loza, A., Gamarra, C. y Condori, N. (2016). Caracterización morfobatimétrica y estimación de sedimentos de la bahía interior de Puno, lago Titicaca, mediante tecnología SIG. Rev. Investig. Altoandin. vol. 18 No. 2, pp 237-248.

[Mor17] Moreno, E., Argota, G., Alfaro, R., Aparicio, M., Atencio, S. y Goyzueta, G. (2017). Determinación interactiva de metales totales en las aguas de la bahía interior del Lago Titicaca- Puno Perú. Rev. Investig. Altoandin. vol. 19, No. 2, pp 125-134.

[Nor92] Northcote, T. (1992). Eutrofización y problemas de polución. El Lago Titicaca, Síntesis del conocimiento limnológico actual. Texto Editores C. Dejoux y A. Iltis .

[Rob10] Roberts, P., Villegas, B. (2010). Hydrodynamic and Water Quality Modeling in Support of the Design of Wastewater Outfalls into Lake Titicaca. Preliminary Report, Prepared for World Bank. 